\title{
Inflammatory demyelinating polyradiculitis in a patient with acute disseminated encephalomyelitis (ADEM)
}

\author{
A Kinoshita, M Hayashi, K Miyamoto, M Oda, H Tanabe
}

\begin{abstract}
A patient with severe acute disseminated encephalomyelitis died 12 days after the first symptom. Necropsy showed widespread severe demyelination in the CNS and some foci of demyelination in the spinal roots. The lesions in the peripheral nervous system were characterised by myelin stripping and the presence of macrophages, being severest in the spinal nerve roots. Some axons were completely demyelinated, whereas the axons themselves were preserved. Pathologically established ongoing demyelination in both CNS and peripheral nervous systems raises the possibility of a shared pathological epitope.
\end{abstract}

$(\mathcal{F}$ Neurol Neurosurg Psychiatry 1996;60:87-90)

Keywords: acute disseminated encephalomyelitis; peripheral nervous system; demyelination

Acute disseminated encephalomyelitis (ADEM) is an immunologically mediated demyelinating disorder that typically affects the white matter of the CNS. Demyelinating lesions of the peripheral nervous system (PNS) have seldom been documented in ADEM, because $\mathrm{ADEM}$ is generally considered to selectively involve the myelin of the CNS.

To our knowledge, there are only three clinical reports in which ADEM has been associated with suspected demyelination of the PNS. ${ }^{1-3}$ Pathological changes in the central and peripheral nervous systems were not, however, confirmed in these cases. We report a case of ADEM in which pathologically established demyelinating polyradiculitis was present in addition to demyelination of the CNS.

Neurology

A Kinoshita

M Hayashi

K Miyamoto

H Tanabe

Department of

Neuropathology, Tokyo

Metropolitan

Neurological Hospital,

Tokyo, Japan

M Oda

Correspondence to:

Dr A Kinoshita, Department

of Neurology, Faculty of

of Neurology, Faculty of

Medicine, Kyoto University,

(

Sakyo-ku, Kyoto, 606-01,

Japan.

Received 20 April 1995

and in revised form

3 August 1995

Accepted 7 September 1995 ter, and with gadolinium-DTPA contrast enhancement was seen in the corpus callosum and left internal capsule. Routine haematological and biochemical tests were normal. A study of CSF on the fifth day in hospital showed slight pleocytosis (10 mononuclear cells $/ \mathrm{mm}^{3}$ ) with a mild increase in protein content (46 $\mathrm{mg} / \mathrm{dl}$ ). The diagnosis was ADEM, and intravenous prednisolone treatment $(60 \mathrm{mg} /$ day $)$ was started. His consciousness level deteriorated rapidly to coma in a few days despite the steroid treatment. He died of brain herniation 10 days after admission.

Materials, methods, and results PATHOLOGICAL FINDINGS: CENTRAL NERVOUS SYSTEM

A general necropsy performed six hours after death showed bilateral uncal herniation with Duret's haemorrhage of the midbrain. No specific abnormalities were noted in the heart, liver, kidney, or other viscera. Routine neuropathological examinations were done on formalin fixed, paraffin embedded samples of the brain and spinal cord. The specimens were stained with haematoxylin-eosin, KlüverBarrera, and Bodian. Diffuse intensive demyelination of the cerebral white matter was found (fig 1). The microscopic findings

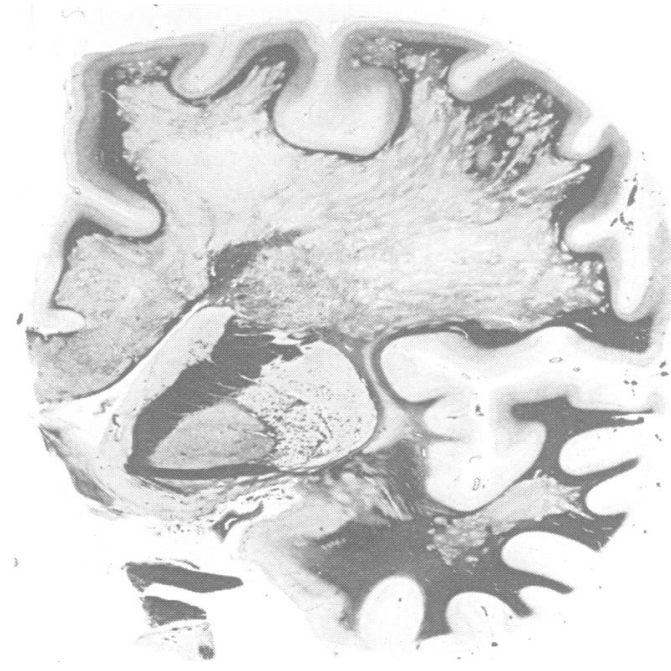

Figure 1 Left cerebral hemisphere. Intensive demyelination in the white matter; patchy in the superficial, and fused and diffuse in the deep regions. The subcortical white matter is relatively spared (Klüver-Barrera). 
Figure 2 Transverse semithin section of a $C 7 A$ fasciculus (toluidine blue $\times$ 780). (A) Arrowheads show demyelinating axons. The myelin sheaths have been stripped from the axolemma, and there is myelin vesiculation and loosening of and

irregularities in the myelin lamella. (B) The same fasciculus as in A. Asterisk: a cluster of infiltrating macrophages. Arrows: a few denuded axons. Widening of the myelin is seen in some fibres (arrowheads).
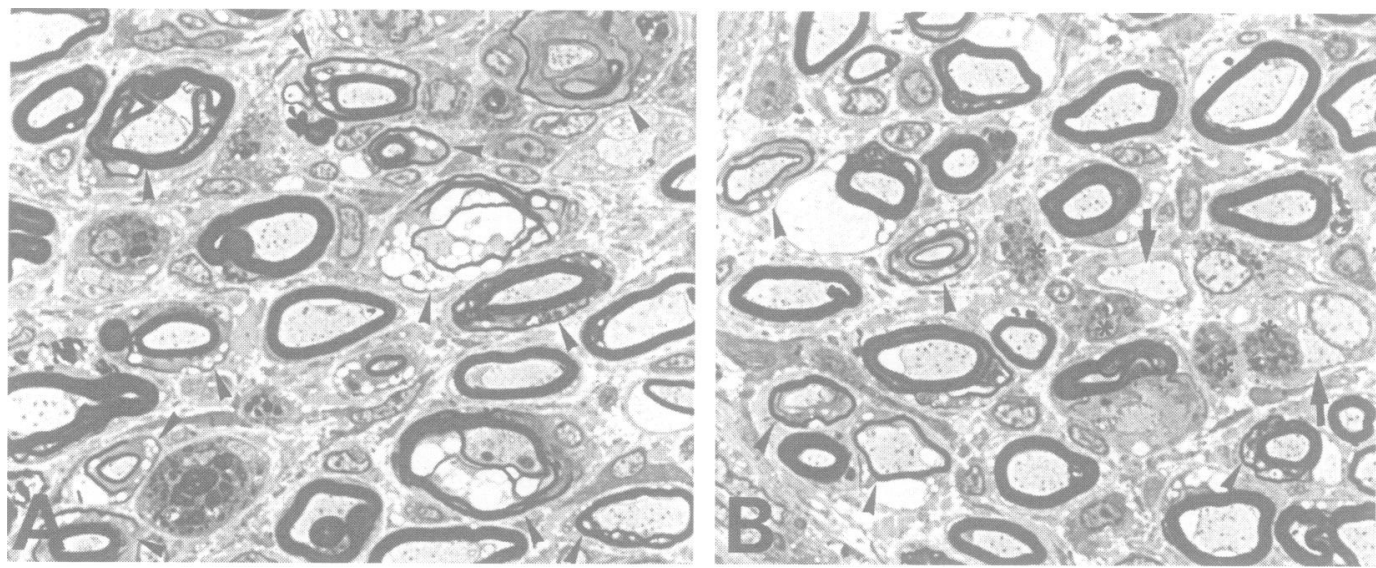

showed many instances of perivascular demyelinating plaque in the cerebral white matter with relative preservation of axons. The plaque was fused in the deep white matter, and numerous lipid laden macrophages were present in those lesions. Perivascular lymphocyte infiltration was present, but not prominent. A few masses of patchy demyelinating plaque were scattered throughout the brainstem, cerebellum, spinal cord, and optic chiasm.

\section{PATHOLOGICAL FINDINGS: PERIPHERAI}

NERVOUS SYSTEM

Morphological analysis was done on the seventh cervical anterior and posterior roots (C7A, C7P), the second thoracic anterior and posterior roots, the 10th thoracic anterior and posterior roots, the brachial plexus, and the median, femoral, and sural nerves. Specimens were fixed in phosphate buffered $2.5 \%$ glutaraldehyde then postfixed in $2 \%$ osmic acid and embedded in Epon. Toluidine blue stained semithin transverse sections were used in the evaluation of these nerves. Uranyl and lead stained ultrathin sections of $\mathrm{C} 7 \mathrm{~A}$ were used for electron microscopy.

A transverse semithin section of a fasciculus of the C7A root (fig 2A) shows fibres with vesiculovacuolar dissolution of the myelin. Varying degrees of myelin breakdown can be seen, from axons with myelin loosening to completely denuded axons. About $30 \%$ of the myelinated fibres in one fasciculus from C7A show widening of the lamellae, some of them associated with intratubal macrophages. Another part of the same C7A fasciculus has several small macrophage foci (fig $2 \mathrm{~B}$ ). The macrophages are stuffed with fragments of myelin debris, lipid droplets, or both. A few denuded axons are surrounded by epineurial macrophages (arrows). Several of the other fibres show widening of the two or three outermost layers (arrowheads), considered an indication of early demyelination. Nearly all the inflammatory cells are macrophages. Extensive sampling of the spinal nerve roots and peripheral nerves showed only minimal inflammatory lymphocytic infiltrates even in lesions with the severest demyelination.

Electron microscopic findings for a C7A fibre showed myelin loosening of its outer layers (fig 3A). The outer lamellae are separated from the rest of the myelin sheath, beneath which there is fine vacuolar material and degenerated myelin. Its axon is almost completely preserved. In another fibre, the myelin is almost completely disrupted, leaving the fibre naked with many vacuoles (fig $3 \mathrm{~B}$ ).

The distribution of active demyelination was patchy, being most conspicuous in the spinal roots. The degree of demyelination varied from fasciculus to fasciculus, some fasciculi from C7A appearing normal with no sign of myelin breakdown or macrophage infiltration. Active demyelination was noted in other spinal roots, but it was of lesser degree than that in C7A. Both the anterior and posterior roots were involved, but the myelin of the anterior roots was more severely damaged. More distal parts of the PNS, such as the brachial plexus and median and femoral nerves, were less involved, having only a few demyelinating fibres. The sural nerve, the most distal nerve examined, was almost normal.
Figure 3 Electron micrographs of a $C 7 A$ fasciculus. $(\times 8000)(A)$ The outer lamella of the myelinated fibre is loosened. The axon is preserved. (bar $=2 \mu \mathrm{m})$ (B) Totally demyelinated fibre in the same fasciculus. (bar $=2 \mu \mathrm{m})$
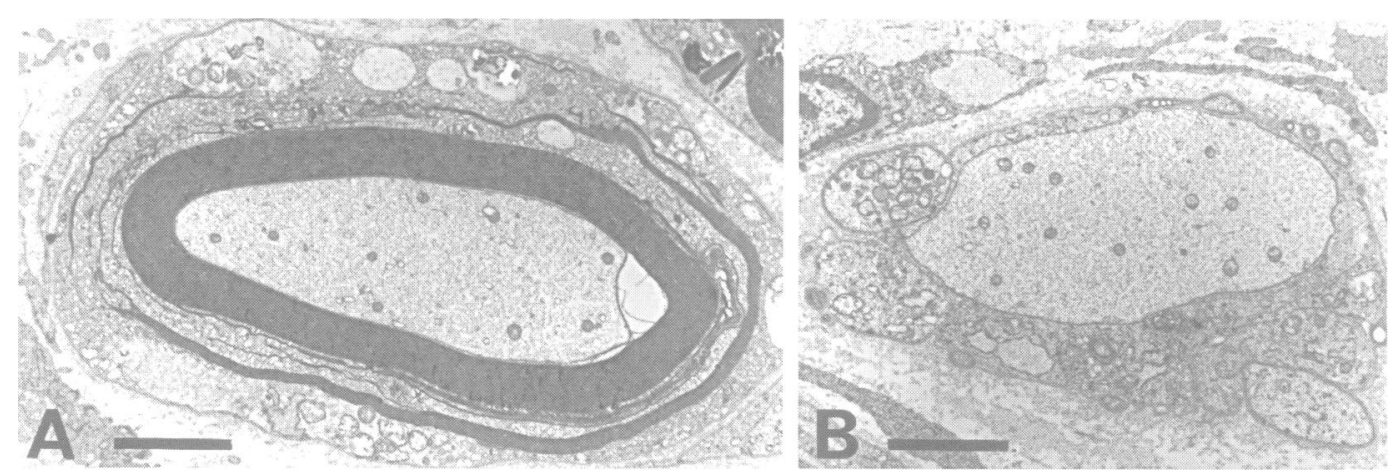


\section{Discussion}

The characteristic feature of the pathological findings for the CNS was disseminated perivascular demyelination which was fused in the deep white matter. No obvious large plaques typical of multiple sclerosis were present. Taking into account the clinical course and these findings, we confirmed the diagnosis of ADEM. This case is unusual because of the extensive amount of demyelination seen in the spinal nerve roots. The pathological examination showed active demyelination in both the CNS and PNS. The principal findings were vesiculovacuolar changes of the myelin and macrophage infiltration. Demyelination was patchy and scattered. Even in the same root, the degree of demyelination varied from fasciculus to fasciculus. Axons were well preserved. The degree of active demyelination was most severe in the spinal roots, but much less in the distal parts of the peripheral nerves. The presence of PNS demyelinating lesions in this patient suggests concomitant polyradiculitis that seems to be similar to the changes for the spinal roots in Guillain-Barré syndrome. . $^{4-6}$

Similar vesicular changes in the myelin were described as the postmortem artifacts in the necropsy materials obtained 20 hours or more after death. ${ }^{7}$ But these changes were never seen in the controls whose necropsy was undertaken two to nine hours after death in our hospital as previously reported, ${ }^{6}$ and the necropsy of this patient was done six hours after death. Moreover, the vesicular changes were often seen where macrophages and denuded axons were observed, but never where they were not seen. Therefore the changes seen in this patient (fig 2A, B, 3A) are considered to be genuine findings rather than artifacts.

Pathological changes in the PNS in ADEM have rarely been studied, perhaps because $\mathrm{ADEM}$ is a disease of acute onset and rapid deterioration with severe CNS involvement; therefore, PNS lesions may be masked by various CNS symptoms. Documented overlap of CNS and PNS demyelinating disorders in ADEM is rare, but demyelinating polyneuropathy has been reported in multiple sclerosis.

In multiple sclerosis, demyelinating lesions have been considered to be localised only in the CNS, but evidence of primary demyelination in the PNS is accumulating. Onion bulb formation, a characteristic feature of chronic demyelination in the PNS, has sometimes been identified in the spinal roots and/or distal nerves of patients with classic multiple sclerosis. ${ }^{8-12}$ On the other hand, in patients with acute multiple sclerosis, Margburg described findings of demyelination of the PNS in his original report, ${ }^{13}$ although the exact nature was not shown. Recently Lassmann et al reported active demyelinating lesions in the spinal roots of a patient with acute multiple sclerosis, who died 12 weeks after onset, and the presence of multiple demarcated large plaques typical of multiple sclerosis. ${ }^{14}$ The patient's tissues showed active demyelinating polyradiculitis with macrophages containing debris. No onion bulb formation was found.

The pathological findings in the PNS for our patient with ADEM showed close similarities with those for the patient of Lassmann et al, ${ }^{14}$ rather than with those for patients with classic multiple sclerosis in that demyelinating lesions were restricted to the spinal roots with myelin stripping and macrophage infiltration and the absence of onion bulb formation. We suspect that these similarities arise from the acuteness of the disease.

Clinically, three cases of ADEM with demyelination of the PNS have been reported so far. ${ }^{1-3}$ In the two reported by Amit et al, ${ }^{1-2}$ there was encephalitis with increased CSF protein and delayed nerve conduction velocities. They suspected that the patients concomitantly had had Guillain-Barré syndrome and ADEM. Nadkarni and Lisak ${ }^{3}$ reported a case in which typical Guillain-Barré syndrome was followed by bilateral optic neuritis after a three week interval. The MRI in that case showed multiple lesions, suggestive of attendant demyelination of the cerebral white matter. Pathological changes in the PNS and CNS, however, were not confirmed in these three cases.

Immune mediated inflammatory demyelinating diseases of the nervous system generally are regarded as selectively involving either central or peripheral myelin; but there are now several reports of combined demyelination of the CNS and PNS as discussed earlier. Why combined demyelination occasionally occurs is not clear, but it suggests the possibility of a shared pathogenic CNS and PNS epitope. Antigenic cross reactivity between the CNS and PNS against myelin proteins and other antigens may elicit similar immune responses, thereby producing demyelination. Our fulminant case of ADEM is evidence of a shared pathogenic CNS and PNS epitope, especially in the spinal roots. To the best of our knowledge, our patient showing PNS involvement in ADEM is the only one in which microscopic and ultrastructural evidence of active demyelination has been documented.

We are particularly grateful to Dr K Yagi (Tokyo Metropolitan Neurological Hospital) and Dr S Kaseda (Minami-Kyusyu National Hospital for their clinical advice, as well as to the Metroplitan Neurolo Metropolitan Neurological Hospital and Tokyo Metropolitan Institute for Neuroscience for their excellent technical assistance.

1 Amit R, Shapira Y, Blank A, Aker M. Acute, severe, central and peripheral nervous system combined demyelination. Pediatr Neurol 1986;2:47-50.

2 Amit R, Glick B, Itzchak Y, Dgani Y, Meyeir S. Acute severe combined demyelination. Childs Nerv Syst 1992, 8:354-6.

3 Nadkarni N, Lisak RP. Guillain-Barré syndrome (GBS) with bilateral optic neuritis and central white matter disease. Neurology 1993;43:842-3.

4 Asbury AK, Arnason BG, Adams RD. The inflammatory lesion in idiopathic polyneuritis. Medicine 1969;48: 173-215.

5 Carpenter $S$. An ultrastructural study of an acute fatal case of the Guillain-Barré syndrome. F Neurol Sci 1972;15: the Guillai $125-40$.

6 Kanda T, Hayashi H, Tanabe H, Tsubaki T, Oda M. A fulminant case of Guillain-Barré syndrome: topographic and fibre size related analysis of demyelinating changes. $\mathcal{f}$ Neurol Neurosurg Psychiatry 1989;52:857-64.

7 Hirano A. Some postmortem structural changes in peripheral myelinated fibers. In: Adachi $M$, Hirano A, Aronson SM, eds. The pathology of the myelinated axon. New York: Igakushoin, 1985:30-48.

8 Ro YI, Alexander CB, Oh SJ. Multiple sclerosis and hypertrophic demyelinating peripheral neuropathy. Muscle Nerve 1983;6:312-6.

9 Rosenberg NL, Bourdette D. Hypertrophic neuropathy and multiple sclerosis. Neurology 1983;33:1361-4. 
10 Rubin M, Karpati G, Carpenter S. Combined central and peripheral myelinopathy. Neurology 1987;37:1287-90.

(n)

"Onion bulb" formations in the cental and periph nervous system in association the central and peripheral nervous system in association with multiple sclerosis and 2 Zee PC, Cohen BA, Walczak T, Jubelt B. Peripheral ner- vous system involvement in multiple sclerosis. Neurology 1991;41:457-60.

13 Marburg O. Die sogennante "acute multiple Sklerose". Fahrbücher für Psychiatrie und Neurologie 1906;7:213-312. 14 Lassmann $\mathrm{H}$, Budka H, Schnaberth G. Inflammatory demyelinating polyradiculitis in a patient with multiple sclerosis. Arch Neurol 1981;38:99-102.

\section{NEUROLOGY IN LITERATURE}

\section{Some Proustian doctors}

Little comment is necessary about these extracts. They perfectly represent the insight which Proust brought into all his writings. If you do not recognise some of your colleagues here, look harder!

Marcel Proust, 1919, Remembrance of things past: within a budding grove

My suffocations having persisted long after any congestion remained that could account for them, my parents brought in a Professor Cottard. It is not enough that a physician who is called in to treat cases of this sort should be learned. Confronted with symptoms which may be those of three or four different complaints, it is in the long run his flair, his instinctive judgment, that must decide with which, despite the more or less similar appearance of them all, he has to deal. This mysterious gift does not imply any superiority in the other departments of the intellect, and a person of the utmost vulgarity, who admires the worst pictures, the worst music, who is without the slightest intellectual curiosity, may perfectly well possess it ...

But Cottard's hesitations were brief and his prescriptions imperious: "Purges, violent and drastic purges; milk for some days, nothing but milk. No meat. No alcohol"... Then, as my health deteriorated, they decided to make me follow Cottard's prescriptions to the letter; in three days my "rattle" and cough had ceased, I could breathe freely ... . and we realised that this imbecile was a great physician.

Marcel Proust, 1920/21, Remembrance of things past: the Guermantes Way

I helped my grandmother into Professor E_-'s lift and a moment later he came to us and took us into his consulting room. But there, pressed for time though he was, his offensive manner changed, such is the force of habit, and his habit was to be friendly, not to say playful, with his patients. Since he knew that my grandmother was a great reader, and was himself one, he devoted the first few minutes to quoting various favourite passages of poetry appropriate to the glorious summer weather. He had placed her in an armchair and himself with his back to the light so as to have a good view of her. His examination was minute and thorough, even obliging me to leave the room for a moment. He continued it after my return, then, having finished, went on, although the quarter of an hour was almost at an end, repeating various quotations to my grandmother. He even made a few jokes which were witty enough, though I should have preferred to hear them on some other occasion, but which completely reassured me by the tone of amusement in which he uttered them ...

"Your grandmother is doomed" he said to me. "It is a stroke brought on by uraemia. In itself, uraemia is not necessarily fatal, but this case seems to be hopeless."

Marcel Proust, 1920/21, Remembrance of things past: the Guermantes Way

My father went to receive him in the drawing-room, like the actor who is next to appear on the stage. He had been sent for not to cure but, to certify, almost in a legal capacity. Dr Dieulafoy may indeed have been a great physician, a marvellous teacher; to the several roles in which he excelled, he added another, in which he remained for forty years without a rival, a role as original as that of the confidant, the clown or the noble father, which consisted in coming to certify that a patient was in extremis. His name alone presaged the dignity with which he would sustain the part, and when the servant announced: " $M$ Dieulafoy," one thought one was in a Molière play. To the dignity of his bearing was added, without being conspicuous, the litheness of a perfect figure. His exaggerated good looks were tempered by a decorum suited to distressing circumstances. In the sable majesty of his frock coat the Professor would enter the room, melancholy without affectation, uttering not one word of condolence that could have been construed as insincere, not being guilty of the slightest infringement of the rules of tact. At the foot of a deathbed it was he and not the Duc de Guermantes who was the great nobleman. Having examined my grandmother without tiring her, and with an excess of reserve which was an act of courtesy to the doctor in charge of the case, he murmured a few words to my father, and bowed respectfully to my mother, to whom I felt that father had positively to restrain himself from saying: "Professor Dieulafoy." But already the latter had turned away, not wishing to seem intrusive, and made a perfect exit, simply accepting the sealed envelope that was slipped into his hand. He did not appear to have seen it, and we ourselves were left wondering for a moment whether we had really given it to him, with such a conjurer's dexterity had he made it vanish without sacrificing one iota of the gravity-which was if anything accentuated-of the eminent consultant in his long frock coat with its silk lapels, his noble features engraved with the most dignified commiseration.

Marcel Proust, 1921/22, Remembrance of things past: cities of the plain

He attached great importance of never being mistaken in his diagnoses ... The reader may perhaps remember that, immediately after my grandmother's stroke, I had taken her to see him, on the afternoon when he was having all his decorations stitched to his coat . . The mistakes made by doctors are innumerable. They err habitually on the side of optimism as to treatment, of pessimism as to the outcome ... Doctors (we do not here include them all, of course, and make a mental reservation of certain admirable exceptions) are in general more displeased, more irritated by the invalidation of their verdicts than pleased by their execution.

Owing to the circumstances of my grandmother's death, the subject interested me, and I had recently read in a book by a great specialist that perspiration was injurious to the kidneys by discharging through the skin something whose proper outlet was elsewhere ... I did not mention this to Dr E___, but of his own accord he said to me: "The advantage of this very hot weather in which perspiration is abundant is that the kidney is correspondingly relieved." Medicine is not an exact science.

G D PERKIN

Regional Neurosciences Centre, Charing Cross Hospital, Fulham Palace Road, London W6 8RF, UK 